\title{
BMJ Open Genome-wide association study of suicide attempt in a Mexican population: a study protocol
}

\author{
Thelma Beatriz González-Castro, ${ }^{1}$ Alma Delia Genis-Mendoza, ${ }^{2}$ \\ Carlos Alfonso Tovilla-Zárate, ${ }^{\circledR 3}$ José Jaime Martínez-Magaña, ${ }^{\circledR 2,4}$ \\ Isela Esther Juárez-Rojop, ${ }^{4}$ Emmanuel Sarmiento, ${ }^{5}$ Humberto Nicolini ${ }^{2}$
}

To cite: González-Castro TB, Genis-Mendoza AD, TovillaZárate CA, et al. Genome-wide association study of suicide attempt in a Mexican population: a study protocol. BMJ Open 2019;9:e25335. doi:10.1136/ bmjopen-2018-025335

- Prepublication history for this paper is available online. To view these files, please visit the journal online (http://dx.doi. org/10.1136/bmjopen-2018025335).

TBG-C and ADG-M contributed equally.

Received 10 July 2018

Revised 25 January 2019

Accepted 12 February 2019
(A) Check for updates

(C) Author(s) (or their employer(s)) 2019. Re-use permitted under CC BY-NC. No commercial re-use. See rights and permissions. Published by BMJ.

For numbered affiliations see end of article.

Correspondence to Dr Humberto Nicolini; hnicolini@inmegen.gob.mx

\section{ABSTRACT}

Introduction Suicidality is a complex behaviour and a major health problem; the specific features that could predispose to suicidal behaviour have been extensively investigated, most frequently in European and Asian populations. Therefore, our aim is to present a protocol that will explore suicide attempt in Mexican individuals diagnosed with psychiatric disorders, through a genomewide association study (GWAS).

Method and analysis We will perform a GWAS by comparing 700 individuals who have suicide attempt history, with control subjects without suicide attempt history $(n=500)$. The genotyping will be conducted using the Infinium PsychArray BeadChip and quality controls will be applied to single nucleotides (SNPs) genotyped. After that, we will perform the imputation using reference panels provided by the Haplotype Reference Consortium. We will perform two different workflows: (A) the classic GWAS analysis applying the same weight to all the variants and $(B)$ an algorithm with prediction of deleteriousness of variants.

Ethics and dissemination This study was approved by the ethics and investigation committees of the National Institute of Genomic Medicine on 22 July 2015, No CEI $215 / 13$. We plan to disseminate research findings in scientific conferences and as a manuscript in peerreviewed journals.

Trial registration number CEI 215/13.

\section{INTRODUCTION}

Suicide is one of the leading causes of death worldwide. Individuals diagnosed with psychiatric disorders have higher rates of suicide compared with the general population, which emphasises a narrow relationship between suicide and psychiatric disorders. In this line of evidence, a considerable majority of suicide victims have had an undiagnosed psychiatric disorder at the time of death. ${ }^{1-3}$ Furthermore, several studies consider psychiatric disorders as one of the main risk factors of suicide. Unfortunately, up to today, the mechanisms of this relationship have not been fully disentangled. ${ }^{45}$

\section{Strengths and limitations of this study}

- This will be the first study that aims to perform a genome-wide association study to assess suicide attempt in a Mexican population.

- The methodological analysis will explore the hypothesis that there are genes that increase the risk of suicide attempt in the Mexican population.

- Recruiting participants from three clinical centres in the country will give a more representative sample of the Mexican population.

- Identifying genetic variants associated with suicide attempt will improve our understanding of the biology underlying this disorder and could be useful for future microarrays designed to study Latin American populations.

- As the Mexican population has a heterogeneous genetic background, a possible limitation of this study will be that heterogeneity might interfere in the interpretation of results.

Suicidal behaviour (SB) has been defined as a complex issue that results from the combination of genetic variants along with personal experiences and environmental contribution; altogether, these factors establish the disease symptomatology manifestation. ${ }^{36}$ The results of studies based on twins, adoptions and families, support the heritability of SB, pinpointing a genetic influence. ${ }^{7-10}$ Subsequently, many candidate-gene association studies have been performed, studying the different phenotypes of SB: suicide attempts, suicide ideation and accomplished suicide. ${ }^{1361112}$ Unfortunately, SB is a polygenic trait and candidate-gene association studies underestimate the genetic background. In an attempt to search for this polygenic variation of $\mathrm{SB}$, other strategies have been developed, such as the genomewide association studies (GWASs). ${ }^{6} 1314$

Over the past decade, a small number of GWASs exploring common genetic variation mostly in suicide attempters have found 
significant associations between genetic components and $\mathrm{SB}$, establishing possible molecular pathways involved in the susceptibility of the disease. ${ }^{1516}$ Although a genetic diathesis model for SB predisposition has been proposed and GWASs have suggested candidate loci or pathways, the only information available comes from studies analysing Caucasian or Asian populations, leaving behind Latin American populations including Mexicans. ${ }^{17-21}$ Therefore, more studies are necessary to have a better comprehension of the SB genetic background.

\section{Objectives}

Our aim is to perform the first GWAS of suicide attempters in a Mexican population, in order to explore and define the involvement of a genetic diathesis that predisposes to SB in this population. In addition, we will explore the hypothesis that there are genes and genetic variants that increase the risk of suicide attempt in the Mexican population and these factors could be common in individuals with a psychiatric diagnostic.

\section{METHODS AND ANALYSIS}

\section{Sample population and setting}

The case group ( $\mathrm{n}=700)$ will be formed by individuals who have had at least one suicide attempt, and this will be determined using the Structured Clinical Interview for Diagnostic and Statistical Manual of Mental Disorders-IV (DSM-IV) (SCID-I and II) in psychiatry outpatient areas from three clinical centres: 'Dr. Gustavo A. Rovirosa' general hospital in Tabasco, 'Dr. Desiderio G. Carbajal' regional hospital in Tabasco and 'Dr. Juan N. Navarro' psychiatric hospital in Mexico City. Individuals used as controls $(n=500)$ will not have current or past history of any $\mathrm{SB}$, will not have first degree relatives with SB history and they will be unrelated to those in the case group. Both cases and controls will be interviewed by two psychiatrist or clinical specialists, together they will determine the presence or absence of suicide attempt. All individuals will be Mexicans with Mexican ascendancy of at least two generations (Mexican parents and grandparents); they will be recruited from several hospitals and outpatient clinics in Mexico.

\section{Patient and public involvement}

The outcomes of the present protocol will be directly communicated to patients who participate or their legal caregivers. The results will also be discussed with their psychiatrists and the corresponding health-education structures of the clinical centres. However, the patients have not directly participated in the study design or any methodological procedures.

\section{Ethics and dissemination}

A written informed consent will be obtained from all individuals who accept to participate. The study will be performed in accordance with the Declaration of Helsinki (59th General Assembly, Seoul, Korea, October
2008). This study has been already approved by the ethics and investigation committees of the National Institute of Genomic Medicine (INMEGEN) on 22 July 2015, No CEI 215/13. We plan to disseminate research findings in scientific conferences and as a manuscript in peer-reviewed journals.

\section{Clinical assessment}

Diagnoses and clinical evaluations will be performed by at least two trained senior psychiatrists of whom at least one of them would have personally examined the patient. All participants will undergo semistructured interviews that will include lifetime and family history of SB, among other clinical features. Suicide attempt will be defined as a self-injurious act that had at least a partial intent to end one's life; the number of attempts, method used and medical damage of past suicide attempts will be gathered.

\section{Genotyping}

DNA will be isolated from peripheral blood leukocytes samples using a standardised protocol of the Genomic Wizard Purification Kit from Promega, as previously reported. ${ }^{22-24}$ The integrity of genetic material will be checked on $1 \%$ agarose gels and quantified by spectrophotometry using the NanoDrop system.

The genotyping will be performed using the Infinium PsychArray BeadChip, following the manufacturer's protocol. ${ }^{25}{ }^{26}$ This array contains approximately 580000 genetic variants, wherein includes a set of genetic variants previously associated with a variety of psychiatric illnesses. All genotyping analyses will be performed at the INMEGEN. For quality controls, we will filter-out samples and variants with call rates lower than $98 \%$ and variants deviating from Hardy-Weinberg equilibrium, with a $\chi^{2} p$ value $<1 \times 10^{-6}$. Gender concordance will be performed based on heterozygosity of $\mathrm{X}$ and $\mathrm{Y}$ chromosomes. All filtering processes will be done using the PLINK V.1.9 software. ${ }^{27}$ Then, we will perform a multidimensional scaling (MDS) analysis in PLINK, all single nucleotides (SNPs) that pass quality controls will be pruned and used to check population stratification in order to evaluate the ancestry of the individuals included. After the MDS analysis, the first five components will be used as covariates in the association analysis. MDS dimensions will be graphically represented using the 'MDS-plot' option. After quality control procedures, we will perform the imputation using reference panels provided by the Haplotype Reference Consortium r.1.1; we will use the Michigan Imputation Server for the imputation. ${ }^{28} 29$

\section{Polygenic risk score calculation}

Polygenic risk score (PRS) is a measurement of genetic liability to schizophrenia, based on the Psychiatric Genomics Consortium (PGC) schizophrenia GWAS. ${ }^{30}$ SNPs will be selected and used in the PRS calculation based on $p$ values obtained in the original PGC GWAS using PRSice. ${ }^{31}$ PRS will be performed to search for suicide attempt associations using linear regression 
models adjusted by age, sex and four MDS components. The estimation of gene-based or set-based association tests using GWAS summary data will be performed using Genome-wide complex trait analysis (GCTA). ${ }^{32} 33$

\section{Statistical analyses}

In order to evaluate the effect of genetic variants on SB, we will perform two workflows: (A) a classic GWAS analysis applying the same importance to all the variants and (B) algorithms for predicting deleteriousness of variants. Concerning the first workflow, we will conduct a mixed linear model analysis on imputed variants with a minor allele frequency of $5 \%$. The implementation of linear mixed models will be performed using the GCTA software. ${ }^{34}$ For the second workflow, we will include the prediction of deliriousness of variants using different prediction algorithms, such as PolyPhen, SIFT, Combined Annotation Dependent Depletion (CADD), Variant Effect Predictor (VEP) and Encyclopedia of DNA Elements (ENCODE) ${ }^{35-39}$ After the functional impact on variants prediction, we will compare cases' allele frequency of loss-of-function, missense with deleterious effect, variants present in regulatory regions and variants with a PHREDCADD score higher than 20, with the allele frequency of populations reported in the 1000 genomes and Exome Aggregation Consortium (ExAC) database. GWAS analysis will be performed on cases with a history of suicide attempt and compared with non-suicide attempters. Genome-wide significance will be set at $\mathrm{p}<5 \times 10^{-8}$.

\section{Power analysis calculation}

For the GWAS analysis, we will perform power calculations using QUANTO V.1.2.4 (http://biostats.usc.edu/ software). This analysis uses a log-additive model of inheritance and is capable of detecting a power of 0.82 at significance threshold $5 \times 10^{-8}$ to detect an effect size of $\mathrm{OR} \geq 1.8$ with a $\mathrm{P}_{0}$ of 0.08 and Minor Allele Frequency (MAF) of 0.25 , as observed in a previous study. ${ }^{14}$ The $\mathrm{P}_{0}$ that we will use is the baseline risk of suicide attempt in our population based on previous reports. ${ }^{40} 41$ Therefore, our study will be powered to detect genetic effects.

\section{DISCUSSION}

Various situations and contexts have been proposed as predictors of SB; among them, the presence of a psychiatric disorder seems to be an important determinant for such behaviour. Likewise, the possible genetic predisposition to manifest SB has been supported by several investigations, but the understanding of the precise genetic system that causes such vulnerability to suicidal tendencies is still largely incomplete. Hence, the principal aim of our protocol study is to explore the potential genetic influence on SB in a Mexican population, throughout a GWAS. In addition, we want to emphasise that to the best of our knowledge, this study protocol will be the first one to evaluate SB in patients with schizophrenia and bipolar disorder in a Mexican population.
The majority of genetic epidemiology evidence suggest that $\mathrm{SB}$ is a complex issue, where there are multiple genes that have a small effect over SB; but if combined, could become predisposing factors. Therefore, association studies that detect small effect contributions can be more useful, which is a strength of this protocol. In this sense, one of the most powerful strengths of the GWAS is that it uses many genetic markers across the whole genome to search for associations with a particular disease; as it is based on no prior assumptions, it explores a large number of genetic variants. For these reasons, we will use GWAS's results to explore the genetic influence on SB in schizophrenics with and without suicide attempt, bipolar patients with and without suicide attempt, suicide attempters and healthy subjects as controls. Therefore, the results of this study will provide information to better comprehend the influence of the genetic background when developing SB, among psychiatric patients.

Additionally, the findings of the present research could provide valuable information for future researchers who attempt to identify genetic risk factors of SB and help to detect and/or treat this disease. Nowadays, there are microarrays that have been used to study several genetic variables in Caucasian and Asian populations; to the best of our knowledge, however, there is no evidence reported of GWAS in studies that evaluate SB in psychiatric patients. Therefore, the outcomes of the current protocol could provide essential information. The use of this type of genetic tools will allow us to identify associated SNPs, missense and insertions and indels in mental illnesses, such as schizophrenia and bipolar disorder, as well as their possible participation as predictors of SB in a Mexican population.

In conclusion, these findings could give important information to improve the design of future chips for molecular diagnosis of psychiatric disorders in Mexicans, which will be very useful in the prevention, diagnosis and prognosis of SB in Mexico. Moreover, the findings will give a better perspective of the genetic background as a predictor of SB in psychiatric diseases. Hence, the outcomes would be useful in genetic research as well as in prevention and early diagnosis of SB in Mexicans.

\section{Author affiliations}

${ }^{1}$ División Académica Multidisciplinaria de Jalpa de Méndez, Universidad Juárez Autónoma de Tabasco, Jalpa de Méndez, Mexico

${ }^{2}$ Laboratorio de Genomica de Enfermedades Psiquiatrica y Neurodegenerativas, Instituto Nacional de Medicina Genomica, Mexico, Mexico

${ }^{3}$ División Académica Multidisciplinaria de Comalcalco, Universidad Juárez Autónoma de Tabasco, División Multidisciplinaria de Comalcalco, Comalcalco, Mexico

${ }^{4}$ División Académica de Ciencias de la Salud, Universidad Juárez Autónoma de Tabasco, Villahermosa, Mexico

${ }^{5}$ Urgencias y Pre-consulta, Hospital Psiquiátrico Infantil Dr. Juan N. Navarro, Ciudad de Mexico, Mexico

Acknowledgements The protocol study will be a doctoral thesis of TBG-C.

Contributors ADG-M, HN and JJM-M conceived the study, participated in its design, helped to draft the manuscript and mentored TBG-C. CAT-Z, ADG-M and JJM-M critically revised successive drafts of the manuscript and provided 
important intellectual input. CAT-Z and TBG-C coordinated and supervised the integration of the manuscript. ES, IEJ-R, HN and ADG-M contributed to developing the analytic plan proposed for this study. All authors read and approved the final manuscript.

Funding This protocol will receive the support of INMEGEN project number 23/2015/I via HN.

Competing interests None declared.

Patient consent for publication Obtained.

Provenance and peer review Not commissioned; externally peer reviewed.

Open access This is an open access article distributed in accordance with the Creative Commons Attribution Non Commercial (CC BY-NC 4.0) license, which permits others to distribute, remix, adapt, build upon this work non-commercially, and license their derivative works on different terms, provided the original work is properly cited, appropriate credit is given, any changes made indicated, and the use is non-commercial. See: http://creativecommons.org/licenses/by-nc/4.0/.

\section{REFERENCES}

1. Perlis $\mathrm{RH}$, Huang J, Purcell S, et al. Genome-wide association study of suicide attempts in mood disorder patients. Am J Psychiatry 2010;167:1499-507.

2. Pulay AJ, Réthelyi JM. Multimarker analysis suggests the involvement of BDNF signaling and microRNA biosynthesis in suicidal behavior. Am J Med Genet B Neuropsychiatr Genet 2016;171:763-76.

3. Mullins N, Perroud N, Uher R, et al. Genetic relationships between suicide attempts, suicidal ideation and major psychiatric disorders: a genome-wide association and polygenic scoring study. Am J Med Genet B Neuropsychiatr Genet 2014;165B:428-37.

4. Perroud N, Uher R, Ng MY, et al. Genome-wide association study of increasing suicidal ideation during antidepressant treatment in the GENDEP project. Pharmacogenomics J 2012;12:68-77.

5. Stein MB, Ware EB, Mitchell C, et al. Genomewide association studies of suicide attempts in US soldiers. Am J Med Genet B Neuropsychiatr Genet 2017;174:786-97.

6. Sokolowski M, Wasserman J, Wasserman D. Rare CNVs in Suicide Attempt include Schizophrenia-Associated Loci and Neurodevelopmental Genes: A Pilot Genome-Wide and FamilyBased Study. PLoS One 2016;11:e0168531.

7. Levine SZ, Goldberg Y, Yoffe R, et al. Suicide attempts in a national population of twins concordant for psychoses. Eur Neuropsychopharmacol 2014;24:1203-9.

8. Linker J, Gillespie NA, Maes H, et al. Suicidal ideation, depression, and conduct disorder in a sample of adolescent and young adult twins. Suicide Life Threat Behav 2012;42:426-36.

9. Petersen L, Sørensen TI, Kragh Andersen P, et al. Genetic and familial environmental effects on suicide attempts: a study of Danish adoptees and their biological and adoptive siblings. $J$ Affect Disord 2014;155:273-7.

10. Roy A, Rylander G, Sarchiapone M. Genetics of suicides. Family studies and molecular genetics. Ann N Y Acad Sci 1997;836:135-57.

11. Mirkovic B, Cohen D, Laurent $C$, et al. A case-control association study of 12 candidate genes and attempted suicide in French adolescents. Int J Adolesc Med Health 2017.

12. Tombácz D, Maróti Z, Kalmár T, et al. High-Coverage Whole-Exome Sequencing Identifies Candidate Genes for Suicide in Victims with Major Depressive Disorder. Sci Rep 2017;7:7106.

13. Gross JA, Bureau A, Croteau J, et al. A genome-wide copy number variant study of suicidal behavior. PLoS One 2015;10:e0128369.

14. Galfalvy $\mathrm{H}$, Haghighi $F$, Hodgkinson $C$, et al. A genome-wide association study of suicidal behavior. Am J Med Genet B Neuropsychiatr Genet 2015;168:557-63.

15. Galfalvy $\mathrm{H}$, Zalsman $\mathrm{G}$, Huang $Y Y$, et al. A pilot genome wide association and gene expression array study of suicide with and without major depression. World J Biol Psychiatry 2013;14:574-82.
16. Laje G, Allen AS, Akula N, et al. Genome-wide association study of suicidal ideation emerging during citalopram treatment of depressed outpatients. Pharmacogenet Genomics 2009;19:666-74.

17. Zai CC, Gonçalves VF, Tiwari AK, et al. A genome-wide association study of suicide severity scores in bipolar disorder. J Psychiatr Res 2015;65:23-9.

18. Menke A, Domschke K, Czamara D, et al. Genome-wide association study of antidepressant treatment-emergent suicidal ideation. Neuropsychopharmacology 2012;37:797-807.

19. Bani-Fatemi A, Graff A, Zai C, et al. GWAS analysis of suicide attempt in schizophrenia: Main genetic effect and interaction with early life trauma. Neurosci Lett 2016;622:102-6.

20. Schosser A, Butler AW, Ising M, et al. Genomewide association scan of suicidal thoughts and behaviour in major depression. PLoS One 2011;6:e20690.

21. Willour VL, Seifuddin F, Mahon PB, et al. A genome-wide association study of attempted suicide. Mol Psychiatry 2012;17:433-44.

22. González-Castro TB, Nicolini H, Lanzagorta N, et al. The role of brain-derived neurotrophic factor (BDNF) Val66Met genetic polymorphism in bipolar disorder: a case-control study, comorbidities, and meta-analysis of 16,786 subjects. Bipolar Disord 2015;17:27-38.

23. González-Castro TB, Tovilla-Zárate C, Juárez-Rojop I, et al. Association of the 5HTR2A gene with suicidal behavior: case-control study and updated meta-analysis. BMC Psychiatry 2013;13:25.

24. González-Castro TB, Tovilla-Zárate CA, Juárez-Rojop I, et al. Association of 5 HTR1A gene variants with suicidal behavior: case-control study and updated meta-analysis. J Psychiatr Res 2013;47:1665-72.

25. Guo Y, He J, Zhao S, et al. Illumina human exome genotyping array clustering and quality control. Nat Protoc 2014;9:2643-62.

26. Borges G, Orozco R, Medina Mora ME. [Risk index for attempted suicide in Mexico]. Salud Publica Mex 2012;54:595-606.

27. Chang CC, Chow CC, Tellier LC, et al. Second-generation PLINK rising to the challenge of larger and richer datasets. Gigascience 2015;4:7.

28. Loh PR, Danecek P, Palamara PF, et al. Reference-based phasing using the Haplotype Reference Consortium panel. Nat Genet 2016;48:1443-8.

29. McCarthy S, Das S, Kretzschmar W, et al. A reference panel of 64,976 haplotypes for genotype imputation. Nat Genet 2016:48:1279-83.

30. Schizophrenia Working Group of the Psychiatric Genomics Consortium. Biological insights from 108 schizophrenia-associated genetic loci. Nature 2014;511:421-7.

31. Euesden J, Lewis CM, O'Reilly PF. PRSice: Polygenic Risk Score software. Bioinformatics 2015;31:1466-8.

32. Yang J, Lee SH, Goddard ME, et al. GCTA: a tool for genome-wide complex trait analysis. Am J Hum Genet 2011;88:76-82.

33. Bakshi A, Zhu Z, Vinkhuyzen AA, et al. Fast set-based association analysis using summary data from GWAS identifies novel gene loci for human complex traits. Sci Rep 2016;6:32894.

34. Yang J, Lee SH, Goddard ME, et al. Genome-wide complex trait analysis (GCTA): methods, data analyses, and interpretations. Methods Mol Biol 2013;1019:215-36.

35. Adzhubei I, Jordan DM, Sunyaev SR. Predicting functional effect of human missense mutations using PolyPhen-2. Curr Protoc Hum Genet 2013; Chapter 7:7.20.1-7.20.41.

36. $\mathrm{Ng} \mathrm{PC}$, Henikoff S. Predicting deleterious amino acid substitutions. Genome Res 2001;11:863-74

37. McLaren W, Gil L, Hunt SE, et al. The Ensembl Variant Effect Predictor. Genome Biol 2016;17:122.

38. ENCODE Project Consortium. An integrated encyclopedia of DNA elements in the human genome. Nature 2012;489:57-74.

39. Kircher M, Witten DM, Jain $\mathrm{P}$, et al. A general framework for estimating the relative pathogenicity of human genetic variants. Nat Genet 2014:46:310-5.

40. Romero-Pimentel AL, Mendoza-Morales RC, Fresan A, et al. Demographic and Clinical Characteristics of Completed Suicides in Mexico City 2014-2015. Front Psychiatry 2018;9:402.

41. Bachmann S. Epidemiology of Suicide and the Psychiatric Perspective. Int J Environ Res Public Health 2018;15:1425. 